УДК 517.53

S. MAJumder, A. DAM

\title{
ON THE TRANSCENDENTAL MEROMORPHIC SOLUTIONS OF A CERTAIN CLASS OF DIFFERENTIAL EQUATIONS
}

\begin{abstract}
S. Majumder, A. Dam. On the transcendental meromorphic solutions of a certain class of differential equations, Mat. Stud. 51 (2019), 130-142.
\end{abstract}

We consider the differential equation

$$
F^{(k)}-a_{2}=e^{\gamma}\left\{\alpha\left(F-a_{1}\right)+\beta\right\},
$$

where $a_{i}(z), \alpha(z)(\not \equiv 0, \infty), \beta(z)(\not \equiv \infty), i=1,2$ are small functions of $F, \gamma$ is an entire function and $k \in \mathbb{N}$. Let $f$ be a transcendental meromorphic function such that $N(r, \infty ; f)=S(r, f)$ and $n \in \mathbb{N}$ such that $n \geq k+1$. If $F=f^{n}$ is a solution of the above differential equation, then

$$
F^{(k)} \equiv \frac{a_{2} \alpha}{a_{1} \alpha-\beta} F
$$

Also we exhibit an example to fortify the condition of our result.

1. Introduction, definitions and results. In the paper a meromorphic function means it is meromorphic in the open complex plane $\mathbb{C}$. We use the standard notations of Nevanlinna theory e.g., $N(r, f), m(r, f), T(r, f), N(r, a ; f), \bar{N}(r, a ; f), m(r, a ; f)$ etc., see [6]. We denote by $S(r, f)$ a quantity, not necessarily the same at each occurrence, that satisfies the condition $S(r, f)=o\{T(r, f)\}$ as $r \rightarrow \infty$ except possibly a set of finite linear measure.

A meromorphic function $a=a(z)$ is called a small function of a meromorphic function $f$, if $T(r, a)=S(r, f)$. Let us denote by $S(f)$ the class of all small functions of $f$. Clearly if $f$ is a transcendental function, then every polynomial is a member of $S(f)$.

Let $f$ and $g$ be two non-constant meromorphic functions and $a \in S(f) \cap S(g)$. If $f-a$ and $g-a$ have the same zeros with the same multiplicities, then we say that $f$ and $g$ share the small function $a \mathrm{CM}$ (counting multiplicities) and if we do not consider the multiplicities, then we say that $f$ and $g$ share the small function $a$ IM (ignoring multiplicities).

Let $k$ be a positive integer and $a \in S(f)$. We use $N_{k)}(r, a ; f)$ to denote the counting function of zeros of $f-a$ with multiplicity not greater than $k, N_{(k+1}(r, a ; f)$ to denote the counting function of zeros of $f-a$ with multiplicity greater than $k$. Similarly we use $\bar{N}_{k)}(r, a ; f)$ and $\bar{N}_{(k+1}(r, a ; f)$ are their respective reduced functions.

In 1996, Brück [1] studied the relation between $f$ and $f^{\prime}$ if an entire function $f$ shares only one finite value $\mathrm{CM}$ with its derivative $f^{\prime}$. In this direction an interesting conjecture was proposed by Brück [1], which is still open in its full generality.

2010 Mathematics Subject Classification: 30D35.

Keywords: meromorphic functions; derivative; small function.

doi:10.15330/ms.51.2.130-142

(C) S. Majumder, A. Dam, 2019 
Conjecture A. Let $f$ be a non-constant entire function. Suppose

$$
\rho_{1}(f):=\limsup _{r \rightarrow \infty} \frac{\log \log T(r, f)}{\log r},
$$

the hyper-order of $f$, is not a positive integer or infinity. If $f$ and $f^{\prime}$ share a finite value $a$ CM, then

$$
\frac{f^{\prime}-a}{f-a}=c
$$

for some $c \in \mathbb{C} \backslash\{0\}$.

The conjecture for the special cases (1) $a=0$ and (2) $N\left(r, 0 ; f^{\prime}\right)=S(r, f)$ had been established by Brück [1]. From the differential equations

$$
\frac{f^{\prime}-a}{f-a}=e^{z^{n}} \text { and } \frac{f^{\prime}-a}{f-a}=e^{e^{z}}
$$

we see that when $\rho_{1}(f)$ is a positive integer or infinity, the conjecture does not hold.

The conjecture for the case where $f$ is of finite order had been proved by Gundersen and Yang [5], the case that $f$ is of infinite order with $\rho_{1}(f)<\frac{1}{2}$ had been proved by Chen and Shon [3]. Recently Cao [2] proved that the Brück conjecture is also true when $f$ is of infinite order with $\rho_{1}(f)=\frac{1}{2}$. But the case $\rho_{1}(f)>\frac{1}{2}$ is still open. However, the corresponding conjecture for meromorphic functions fails in general (see [5]). For example, if

$$
f(z)=\frac{2 e^{z}+z+1}{e^{z}+1}
$$

then $f$ and $f^{\prime}$ share $1 \mathrm{CM}$, but (1) does not hold.

It is interesting to ask what happens if $f$ is replaced by a power of it, say, $f^{n}$ in Brück's conjecture. From (3) we see that the conjecture does not hold without any restriction on the hyper-order when $n=1$. So we only need to focus on the problem when $n \geq 2$.

Perhaps Yang and Zhang [9] were the first to consider the uniqueness of a power of an entire function $F=f^{n}$ and its derivative $F^{\prime}$ when they share certain value and that leads to a specific form of the function $f$.

Yang and Zhang [9] proved that the Brück conjecture holds for the function $f^{n}$ and the order restriction on $f$ is not needed if $n$ is relatively large. Actually they proved the following result.

Theorem A ([9]). Let $f$ be a non-constant entire function, $n \in \mathbb{N}$ such that $n(\geq 7)$ and let $F=f^{n}$. If $F$ and $F^{\prime}$ share $1 C M$, then $F \equiv F^{\prime}$ and $f(z)$ assumes the form $f(z)=c e^{\frac{1}{n} z}$, where $c \in \mathbb{C} \backslash\{0\}$.

Improving all the results obtained in [9], Zhang [11] proved the following theorem.

Theorem B ([11]). Let $f$ be a non-constant entire function, $k, n \in \mathbb{N}$ and $a(z)(\not \equiv 0, \infty) \in$ $S(f)$. If $f^{n}-a$ and $\left(f^{n}\right)^{(k)}-a$ share $0 C M$ and $n \geq k+5$, then $f^{n} \equiv\left(f^{n}\right)^{(k)}$ and $f(z)$ assumes the form $f(z)=c e^{\frac{\lambda}{n} z}$, where $c \in \mathbb{C} \backslash\{0\}$ and $\lambda^{k}=1$.

In 2009, Zhang and Yang [12] further improved the above result in the following manner. 
Theorem $\mathbf{C}([12])$. Let $f$ be a non-constant entire function, $k, n \in \mathbb{N}$ and $a(z)(\not \equiv 0, \infty) \in$ $S(f)$. Suppose $f^{n}-a$ and $\left(f^{n}\right)^{(k)}-a$ share $0 C M$ and $n \geq k+2$. Then conclusion of Theorem $B$ holds.

In 2010, Zhang and Yang [13] further improved Theorem C in the following direction.

Theorem D ([13]). Let $f$ be a non-constant entire function and $k, n \in \mathbb{N}$. Suppose $f^{n}$ and $\left(f^{n}\right)^{(k)}$ share $1 C M$ and $n \geq k+1$. Then conclusion of Theorem $B$ holds.

In 2011, Lü and Yi [7] proved the following extension of Theorem D.

Theorem $\mathbf{E}([7])$. Let $f$ be a transcendental entire function, $k, n \in \mathbb{N}$ such $n \geq k+1$, $F=f^{n}$ and $Q \not \equiv 0$ be a polynomial. If $F-Q$ and $F^{(k)}-Q$ share $0 C M$, then $F \equiv F^{(k)}$ and $f(z)=c e^{w z / n}$, where $c$ and $w \in \mathbb{C} \backslash\{0\}$ such that $w^{k}=1$.

Remark 1. It is easy to see that the condition $n \geq k+1$ in Theorem $\mathrm{E}$ is sharp by the following example.

Example 1. Let $f(z)=e^{e^{z}} \int_{0}^{z} e^{-e^{t}}\left(1-e^{t}\right) t d t$ and $n=1, k=1$. Then

$$
\frac{f^{\prime}(z)-z}{f(z)-z}=e^{z}
$$

and $f^{\prime}(z)-z$ and $f(z)-z$ share $0 \mathrm{CM}$, but $f^{\prime} \not \equiv f$.

Now observing the above theorem, Lü, Li and Yang [8] asked the following question:

Question 1. What can be said "if $f^{n}-Q_{1}$ and $\left(f^{n}\right)^{(k)}-Q_{2}$ share the value 0 CM", where $Q_{1}$ and $Q_{2}$ are polynomials, and $Q_{1} Q_{2} \not \equiv 0$ ?

Lü, Li and Yang [8] answered the above question for $k=1$ by giving the transcendental entire solutions of the equation

$$
F^{\prime}-Q_{1}=R e^{\alpha}\left(F-Q_{2}\right)
$$

where $F=f^{n}, R$ is a rational function and $\alpha$ is an entire function and they obtained the following result.

Theorem $\mathbf{F}([8])$. Let $f$ be a transcendental entire function and let $F=f^{n}$ be a solution of equation (3), $n \in \mathbb{N}$ such that $n \geq 2$, then $\frac{Q_{1}}{Q_{2}}$ is a polynomial and $f^{\prime} \equiv \frac{Q_{1}}{n Q_{2}} f$.

We now pose the following questions as open problems.

Question 2. Is Theorem F true for equation (3) with $F^{(k)}$ instead $F$ ?

Question 3. Is Theorem F valid for a transcendental meromorphic function?

Question 4. What are properties of the function $f$ or $F$ if $Q_{1}, Q_{2}$ and $R$ in Theorem $\mathrm{F}$ are replaced by small functions of $f$ ?

Now our objective to write this paper is to solve the above questions. The following theorem is the main result in this paper. 
Theorem 1. Let $f$ be a transcendental meromorphic function such that $N(r, \infty ; f)=S(r, f)$ and $a_{i}(z), \alpha(z)(\not \equiv 0, \infty) \in S(f), \beta(z)(\not \equiv \infty) \in S(f)$, where $i=1,2$. Let $k, n \in \mathbb{N}$ such that $n \geq k+1$. If $F=f^{n}$ is a solution of the equation

$$
F^{(k)}-a_{2}=e^{\gamma}\left\{\alpha\left(F-a_{1}\right)+\beta\right\},
$$

where $\gamma$ is an entire function, then

$$
F^{(k)} \equiv \frac{a_{2} \alpha}{a_{1} \alpha-\beta} F
$$

From Theorem 1 we have the following corollary.

Corollary 1. Let $f$ be a transcendental meromorphic function such that $N(r, \infty ; f)=$ $S(r, f)$ and $a_{i}(z), \alpha(z)(\not \equiv 0, \infty) \in S(f)$, where $i=1,2$. Let $k, n \in \mathbb{N}$ such that $n \geq k+1$. If $F=f^{n}$ is a solution of the equation

$$
F^{(k)}-a_{2}=\alpha e^{\gamma}\left(F-a_{1}\right),
$$

where $\gamma$ is an entire function, then

$$
F^{(k)} \equiv \frac{a_{2}}{a_{1}} F
$$

Remark 2. It is easy to see that the condition $n \geq k+1$ in Theorem 1 is sharp by the following example.

Example 2. Let

$$
f(z)=e^{e^{z^{2}}}+1
$$

where

$a_{1}(z)=1-\frac{1}{2\left(1+e^{z^{2}}\right)}, \quad a_{2}(z)=-\frac{2 z}{1+e^{-z^{2}}}, \quad \alpha(z)=2 z, \quad \beta(z)=\frac{z}{1+e^{z^{2}}}$ and $\gamma(z)=z^{2}$.

Clearly $a_{1}, a_{2}, \alpha, \beta \in S(f)$. Note that

$$
f^{\prime}(z)=2 z e^{z^{2}} e^{e^{z^{2}}}
$$

Therefore

$$
\begin{gathered}
e^{\gamma(z)}\left\{\alpha(z)\left(f(z)-a_{1}(z)\right)+\beta(z)\right\}=\frac{2 z}{e^{-z^{2}}+1}\left[\left(e^{z^{2}}+1\right) e^{e^{z^{2}}}+1\right] \\
f^{\prime}(z)-a_{2}(z)=\frac{2 z}{1+e^{-z^{2}}}\left[\left(e^{z^{2}}+1\right) e^{e^{z^{2}}}+1\right] .
\end{gathered}
$$

Thus

$$
f^{\prime}(z)-a_{2}(z)=e^{\gamma(z)}\left\{\alpha(z)\left(f(z)-a_{1}(z)\right)+\beta(z)\right\}
$$

but

$$
\frac{a_{2}(z) \alpha(z)}{a_{1}(z) \alpha(z)-\beta(z)} f(z)=-2 z f(z) \not \equiv f^{\prime}(z) .
$$


2. Lemmas. In this section we present the lemmas which will be needed in the sequel.

Lemma 1 ([4]). Suppose that $f$ is a transcendental meromorphic function and that

$$
f^{n}(z) P(f(z))=Q(f(z)),
$$

where $P(f(z))$ and $Q(f(z))$ are differential polynomials in $f$ with functions of small proximity related to $f$ as the coefficients and the degree of $Q(f(z))$ is at most $n$. Then $m(r, P)=S(r, f)$.

Lemma 2 ([6]). Let $f$ be a non-constant meromorphic function and let $a_{1}(z), a_{2}(z)$ be two meromorphic functions such that $a_{i} \in S(f), i=1,2$. Then

$$
T(r, f) \leq \bar{N}(r, \infty ; f)+\bar{N}\left(r, a_{1} ; f\right)+\bar{N}\left(r, a_{2} ; f\right)+S(r, f) .
$$

Lemma 3 ([10]). Let $f$ be a non-constant meromorphic function and let $a_{n}(z)(\not \equiv 0), a_{n-1}(z)$, $\ldots, a_{0}(z) \in S(f)$. Then $T\left(r, \sum_{i=0}^{n} a_{i} f^{i}\right)=n T(r, f)+S(r, f)$.

\section{Proof of the theorem.}

Proof of Theorem 1. Let

$$
F=f^{n} \text {. }
$$

By the given condition we have

$$
e^{\gamma}=\frac{F^{(k)}-a_{2}}{\alpha\left(F-a_{1}\right)+\beta} \text {, i.e., } F^{(k)}-a_{2}=e^{\gamma}\left\{\alpha\left(F-a_{1}\right)+\beta\right\} .
$$

Note that

$$
\begin{aligned}
& T\left(r, e^{\gamma}\right) \leq T\left(r, F^{(k)}-a_{2}\right)+T\left(r, \alpha\left(F-a_{1}\right)+\beta\right) \leq T\left(r, F^{(k)}\right)+T(r, F)+S(r, F)+ \\
& \quad+S(r, f) \leq(k+1) T\left(r, f^{n}\right)+n T(r, f)+S(r, f)=n(k+2) T(r, f)+S(r, f),
\end{aligned}
$$

which implies that $S\left(r, e^{\gamma}\right)$ can be replaced by $S(r, f)$. Also we see that

$$
T\left(r, \gamma^{\prime}\right)=m\left(r, \gamma^{\prime}\right)=m\left(r, \frac{\left(e^{\gamma}\right)^{\prime}}{e^{\gamma}}\right)=S\left(r, e^{\gamma}\right)
$$

and so $\gamma^{\prime} \in S(f)$. Let $\xi=\frac{\alpha^{\prime}}{\alpha}+\gamma^{\prime}$. Therefore

$$
T(r, \xi)=T\left(r, \frac{\alpha^{\prime}}{\alpha}+\gamma^{\prime}\right) \leq T\left(r, \frac{\alpha^{\prime}}{\alpha}\right)+T\left(r, \gamma^{\prime}\right)=S(r, f)
$$

and so $\xi \in S(f)$. Differentiating (5) once we get

$$
F^{(k+1)}-a_{2}^{\prime}=e^{\gamma} \gamma^{\prime}\left\{\alpha\left(F-a_{1}\right)+\beta\right\}+e^{\gamma}\left\{\alpha^{\prime}\left(F-a_{1}\right)+\alpha\left(F^{\prime}-a_{1}^{\prime}\right)+\beta^{\prime}\right\} .
$$

Now dividing (6) by (5), we have

$$
\begin{gathered}
\frac{F^{(k+1)}-a_{2}^{\prime}}{F^{(k)}-a_{2}}=\gamma^{\prime}+\frac{\alpha^{\prime}\left(F-a_{1}\right)+\alpha\left(F^{\prime}-a_{1}^{\prime}\right)+\beta^{\prime}}{\alpha\left(F-a_{1}\right)+\beta}, \\
\alpha F^{(k+1)} F-\left(a_{1} \alpha-\beta\right) F^{(k+1)}-a_{2}^{\prime} \alpha F+a_{1} a_{2}^{\prime} \alpha-a_{2}^{\prime} \beta=
\end{gathered}
$$




$$
\begin{gathered}
=\gamma^{\prime}\left(F^{(k)}-a_{2}\right)\left\{\alpha\left(F-a_{1}\right)+\beta\right\}+\alpha^{\prime}\left(F-a_{1}\right)\left(F^{(k)}-a_{2}\right) \\
+\alpha\left(F^{(k)}-a_{2}\right)\left(F^{\prime}-a_{1}^{\prime}\right)+\beta^{\prime}\left(F^{(k)}-a_{2}\right), \\
\alpha F^{(k+1)} F-\left(\alpha^{\prime}+\alpha \gamma^{\prime}\right) F^{(k)} F-\alpha F^{(k)} F^{\prime}= \\
=\left(a_{1} \alpha-\beta\right) F^{(k+1)}-\left(a_{1} \alpha^{\prime}+a_{1}^{\prime} \alpha-\beta^{\prime}+a_{1} \alpha \gamma^{\prime}-\beta \gamma^{\prime}\right) F^{(k)}-a_{2} \alpha F^{\prime}+ \\
+\left(a_{2}^{\prime} \alpha-a_{2} \alpha \gamma^{\prime}-a_{2} \alpha^{\prime}\right) F+a_{1} a_{2} \alpha \gamma^{\prime}+a_{1} a_{2} \alpha^{\prime}-a_{1} a_{2}^{\prime} \alpha+a_{1}^{\prime} a_{2} \alpha-a_{2} \beta \gamma^{\prime}-a_{2} \beta^{\prime}+a_{2}^{\prime} \beta
\end{gathered}
$$

and so

$$
\begin{gathered}
F^{(k+1)} F-\xi F^{(k)} F-F^{(k)} F^{\prime}= \\
=\frac{1}{\alpha}\left\{\left(a_{1} \alpha-\beta\right) F^{(k+1)}-\left(a_{1} \alpha^{\prime}+a_{1}^{\prime} \alpha-\beta^{\prime}+a_{1} \alpha \gamma^{\prime}-\beta \gamma^{\prime}\right) F^{(k)}-a_{2} \alpha F^{\prime}+\right. \\
\left.+\left(a_{2}^{\prime} \alpha-a_{2} \alpha \gamma^{\prime}-a_{2} \alpha^{\prime}\right) F+a_{1} a_{2} \alpha \gamma^{\prime}+a_{1} a_{2} \alpha^{\prime}-a_{1} a_{2}^{\prime} \alpha+a_{1}^{\prime} a_{2} \alpha-a_{2} \beta \gamma^{\prime}-a_{2} \beta^{\prime}+a_{2}^{\prime} \beta\right\} .
\end{gathered}
$$

Now we consider following two cases.

Case 1. Suppose $F^{(k+1)} F-\xi F^{(k)} F-F^{(k)} F^{\prime} \not \equiv 0$.

Immediately we have following two subcases.

Subcase 1.1. Suppose $n>k+1$. By induction we deduce from (4) that

$$
F^{(k)}=\sum_{\lambda} A_{1 \lambda} f^{l_{0}^{\lambda}}\left(f^{\prime}\right)^{l_{1}^{\lambda}} \ldots\left(f^{(k)}\right)^{l_{k}^{\lambda}},
$$

where $l_{0}^{\lambda}, l_{1}^{\lambda}, \ldots, l_{k}^{\lambda}$ are non-negative integers satisfying

$$
\sum_{j=0}^{k} l_{j}^{\lambda}=n, n-k \leq l_{0}^{\lambda} \leq n-1
$$

and $A_{1 \lambda}$ are constants and

$$
F^{(k+1)}=\sum_{\lambda} B_{1 \lambda} f^{p_{0}^{\lambda}}\left(f^{\prime}\right)^{p_{1}^{\lambda}} \ldots\left(f^{(k+1)}\right)^{p_{k+1}^{\lambda}}
$$

where $p_{0}^{\lambda}, p_{1}^{\lambda}, \ldots, p_{k+1}^{\lambda}$ are non-negative integers satisfying $\sum_{j=0}^{k+1} p_{j}^{\lambda}=n, n-k-1 \leq p_{0}^{\lambda} \leq n-1$, i.e., $0 \leq p_{0}^{\lambda} \leq n-1$ and $B_{1 \lambda}$ are constants. Note that

$$
F^{(k)}=f^{n-k-1} \sum_{\lambda} A_{1 \lambda} f^{l_{0}^{\lambda}-(n-k-1)}\left(f^{\prime}\right)^{l_{1}^{\lambda}} \ldots\left(f^{(k)}\right)^{l_{k}^{\lambda}}
$$

where $1 \leq l_{0}^{\lambda}-(n-k-1) \leq k$ and

$$
F^{(k+1)}=f^{n-k-1} \sum_{\lambda} B_{1 \lambda} f^{p_{0}^{\lambda}-(n-k-1)}\left(f^{\prime}\right)^{p_{1}^{\lambda}} \ldots\left(f^{(k+1)}\right)^{p_{k+1}^{\lambda}},
$$

where $0 \leq p_{0}^{\lambda}-(n-k-1) \leq k$. Now from (4), (8) and (9), we get

$$
F^{(k+1)} F-\xi F^{(k)} F-F^{(k)} F^{\prime}=f^{n} f^{n-k-1} P(f),
$$

where

$$
P(f)=\sum_{\lambda} B_{1 \lambda} f^{p_{0}^{\lambda}-(n-k-1)}\left(f^{\prime}\right)^{p_{1}^{\lambda}} \ldots\left(f^{(k+1)}\right)^{p_{k+1}^{\lambda}-}
$$




$$
-\xi \sum_{\lambda} A_{1 \lambda} f^{l_{0}^{\lambda}-(n-k-1)}\left(f^{\prime}\right)^{l_{1}^{\lambda}} \ldots\left(f^{(k)}\right)^{l_{k}^{\lambda}}-n \sum_{\lambda} A_{1 \lambda} f^{l_{0}^{\lambda}-(n-k)}\left(f^{\prime}\right)^{l_{1}^{\lambda}+1} \ldots\left(f^{(k)}\right)^{l_{k}^{\lambda}}
$$

is a differential polynomial in $f$ of degree $k+1$. In particular every monomial of $P(f)$ is of the form

$$
R_{\lambda}\left(\alpha, \alpha^{\prime}, \gamma^{\prime}\right) f^{q_{0}^{\lambda}}\left(f^{\prime}\right)^{q_{1}^{\lambda}} \ldots\left(f^{(k+1)}\right)^{q_{k+1}^{\lambda}}
$$

where $q_{0}^{\lambda}, \ldots, q_{k+1}^{\lambda}$ are non-negative integers satisfying $\sum_{j=0}^{k+1} q_{j}^{\lambda}=k+1$ and $q_{0}^{\lambda} \leq k$, $R_{\lambda}\left(\alpha, \alpha^{\prime}, \gamma^{\prime}\right)$ is a polynomial in $\alpha, \alpha^{\prime}$ and $\gamma^{\prime}$ with constant coefficients. Now from (7) and (10) we have

$$
f^{n} f^{n-k-1} P(f)=Q(f),
$$

where

$$
\begin{gathered}
Q(f)=\frac{1}{\alpha}\left\{\left(a_{1} \alpha-\beta\right) F^{(k+1)}-\left(a_{1} \alpha^{\prime}+a_{1}^{\prime} \alpha-\beta^{\prime}+a_{1} \alpha \gamma^{\prime}-\beta \gamma^{\prime}\right) F^{(k)}-a_{2} \alpha F^{\prime}+\right. \\
\left.+\left(a_{2}^{\prime} \alpha-a_{2} \alpha \gamma^{\prime}-a_{2} \alpha^{\prime}\right) F+a_{1} a_{2} \alpha \gamma^{\prime}+a_{1} a_{2} \alpha^{\prime}-a_{1} a_{2}^{\prime} \alpha+a_{1}^{\prime} a_{2} \alpha-a_{2} \beta \gamma^{\prime}-a_{2} \beta^{\prime}+a_{2}^{\prime} \beta\right\}
\end{gathered}
$$

is a differential polynomial in $f$ of degree $n$. Since $f^{n} f^{n-k-1} P(f) \equiv F^{(k+1)} F-\xi F^{(k)} F-$ $F^{(k)} F^{\prime} \not \equiv 0$, it follows that $P(f) \not \equiv 0$. Now from Lemma 1 and (11) we have

$$
m(r, \infty ; P(f))=S(r, f) \text { and } m\left(r, \infty ; f^{n-k-1} P(f)\right)=S(r, f) .
$$

Since $N(r, \infty ; f)=S(r, f)$, we have

$$
P(f) \in S(f) \text { and } f^{n-k-1} P(f) \in S(f) .
$$

Note that

$$
\begin{gathered}
m\left(r, \infty ; f^{n-k-1}\right)=m\left(r, \infty ; f^{n-k-1} P(f) \frac{1}{P(f)}\right) \leq m\left(r, \infty ; f^{n-k-1} P(f)\right)+m\left(r, \infty ; \frac{1}{P(f)}\right) \leq \\
\leq S(r, f)+T(r, P(f))=S(r, f),
\end{gathered}
$$

i.e., $m(r, \infty ; f)=S(r, f)$. Therefore $T(r, f)=N(r, \infty ; f)+m(r, \infty ; f)=S(r, f)$, which is impossible.

Subcase 1.2. Suppose $n=k+1$. Now from (4) we deduce that

$$
\begin{gathered}
F^{(k)}=\frac{d^{k}}{d z^{k}}\left\{f^{k+1}\right\}=\frac{d^{k-1}}{d z^{k-1}}\left\{(k+1) f^{k} f^{\prime}\right\}=(k+1) \frac{d^{k-2}}{d z^{k-2}}\left\{k f^{k-1}\left(f^{\prime}\right)^{2}+f^{k} f^{\prime \prime}\right\}= \\
=(k+1) k \frac{d^{k-2}}{d z^{k-2}}\left\{f^{k-1}\left(f^{\prime}\right)^{2}\right\}+(k+1) \frac{d^{k-2}}{d z^{k-2}}\left\{f^{k} f^{\prime \prime}\right\}= \\
=(k+1) k \frac{d^{k-3}}{d z^{k-3}}\left\{(k-1) f^{k-2}\left(f^{\prime}\right)^{3}\right\}+(k+1) k \frac{d^{k-3}}{d z^{k-3}}\left\{2 f^{k-1} f^{\prime} f^{\prime \prime}\right\}+ \\
\quad+(k+1) \frac{d^{k-3}}{d z^{k-3}}\left\{k f^{k-1} f^{\prime} f^{\prime \prime}\right\}+(k+1) \frac{d^{k-3}}{d z^{k-3}}\left\{f^{k} f^{\prime \prime \prime}\right\}= \\
=(k+1) k(k-1) \frac{d^{k-3}}{d z^{k-3}}\left\{f^{k-2}\left(f^{\prime}\right)^{3}\right\}+2(k+1) k \frac{d^{k-3}}{d z^{k-3}}\left\{f^{k-1} f^{\prime} f^{\prime \prime}\right\}+ \\
+(k+1) k \frac{d^{k-3}}{d z^{k-3}}\left\{f^{k-1} f^{\prime} f^{\prime \prime}\right\}+(k+1) \frac{d^{k-3}}{d z^{k-3}}\left\{f^{k} f^{\prime \prime \prime}\right\}=\ldots . .
\end{gathered}
$$




$$
=(k+1) ! f\left(f^{\prime}\right)^{k}+\frac{k(k-1)}{4}(k+1) ! f^{2}\left(f^{\prime}\right)^{k-2} f^{\prime \prime}+\ldots+(k+1) f^{k} f^{(k)} .
$$

Therefore

$$
\frac{f^{\prime}}{f} F^{(k)}=(k+1) !\left(f^{\prime}\right)^{k+1}+\frac{k(k-1)}{4}(k+1) ! f\left(f^{\prime}\right)^{k-1} f^{\prime \prime}+\ldots+(k+1) f^{k-1} f^{\prime} f^{(k)}
$$

and

$$
F^{(k+1)}=(k+1) !\left(f^{\prime}\right)^{k+1}+\frac{k(k+1)}{2}(k+1) ! f\left(f^{\prime}\right)^{k-1} f^{\prime \prime}+\ldots+(k+1) f^{k} f^{(k+1)} .
$$

Putting (4), (12), (13) and (14) into (7), we have

$$
f^{n} P(f)=Q(f)
$$

where $Q(f)$ is a differential polynomial in $f$ of degree $n$ and

$$
\begin{aligned}
& P(f)=F^{(k+1)}-\xi F^{(k)}-n \frac{f^{\prime}}{f} F^{(k)}=-k(k+1) !\left(f^{\prime}\right)^{k+1}-(k+1) ! \xi f\left(f^{\prime}\right)^{k}+ \\
&+\frac{k(k+1)(3-k)(k+1) !}{4} f\left(f^{\prime}\right)^{k-1} f^{\prime \prime}+\ldots+(k+1) f^{k} f^{(k+1)}- \\
&-(k+1) \xi f^{k} f^{(k)}-(k+1)^{2} f^{k-1} f^{\prime} f^{(k)}=-k(k+1) !\left(f^{\prime}\right)^{k+1}+R_{1}(f),
\end{aligned}
$$

is a differential polynomial in $f$ of degree $k+1$ and $R_{1}(f)$ is a differential polynomial in $f$ such that each term of $R_{1}(f)$ contains $f^{m}$ for some $m(1 \leq m \leq n-1)$ as a factor.

Since $f^{n} P(f) \equiv F^{(k+1)} F-\xi F^{(k)} F-F^{(k)} F^{\prime} \not \equiv 0$, it follows that $P(f) \not \equiv 0$. Then by Lemma 1 we get $m(r, \infty ; P)=S(r, f)$. Since $N(r, \infty ; f)=S(r, f)$, we have

$$
P(f) \in S(f) \text { and } P^{\prime}(f) \in S(f) .
$$

Let $z_{1}$ be a zero of $f$ with multiplicity $p_{1}(\geq 2)$. Then from (16) we see that $z_{1}$ is a zero of $P(f)$ with multiplicity $\left(p_{1}-1\right)(k+1) \geq 2\left(p_{1}-1\right) \geq p_{1}$. Consequently we have

$$
N_{(2}(r, 0 ; f) \leq N(r, 0 ; P(f)) \leq T(r, P(f))=S(r, f) .
$$

Also from (16) we have

$$
m\left(r, \frac{P(f)}{f^{k+1}}\right)=S(r, f)
$$

Consequently

$$
m\left(r, \frac{1}{f^{k+1}}\right) \leq m\left(r, \frac{P(f)}{f^{k+1}}\right)+m\left(r, \frac{1}{P(f)}\right) \leq T(r, P(f))+S(r, f)=S(r, f),
$$

i.e., $m\left(r, \frac{1}{f}\right)=S(r, f)$. Therefore

$$
T(r, f)=N(r, 0 ; f)+m\left(r, \frac{1}{f}\right)=N_{1)}(r, 0 ; f)+S(r, f) .
$$

Note that from (16) we get

$$
P^{\prime}(f)=A_{1}\left(f^{\prime}\right)^{k} f^{\prime \prime}+B_{1}\left(f^{\prime}\right)^{k+1}+S_{1}(f),
$$


is a differential polynomial in $f$, where $A_{1}=-\frac{1}{4} k(k+1)^{2}(k+1)$ !, $B_{1}=-(k+1)$ ! $\xi$ and $S_{1}(f)$ is a differential polynomial in $f$ such that each term of $S_{1}(f)$ contains $f^{m}$ for some $m$ $(1 \leq m \leq n-1)$ as a factor.

Let $z_{2}$ be a simple zero of $f$. Then from (16) and (19) we have $P\left(f\left(z_{2}\right)\right)=-k(k+1) !\left(f^{\prime}\left(z_{2}\right)\right)^{k+1}$ and $P^{\prime}\left(f\left(z_{2}\right)\right)=A_{1}\left(f^{\prime}\left(z_{2}\right)\right)^{k} f^{\prime \prime}\left(z_{2}\right)+B_{1}\left(z_{2}\right)\left(f^{\prime}\left(z_{2}\right)\right)^{k+1}$.

This shows that $z_{2}$ is a zero of $P(f) f^{\prime \prime}-\left[K_{1} P^{\prime}(f)-K_{2} P(f)\right] f^{\prime}$, where $K_{1}=\frac{-k(k+1) !}{A_{1}}$ and $K_{2}=\frac{B_{1}}{A_{1}}$. Also we see that $K_{1} \in S(f)$ and $K_{2} \in S(f)$. Let

$$
\Phi_{1}=\frac{P(f) f^{\prime \prime}-\left[K_{1} P^{\prime}(f)-K_{2} P(f)\right] f^{\prime}}{f} .
$$

Suppose $\Phi_{1}(z) \not \equiv 0$. Then clearly $m\left(r, \Phi_{1}\right)=S(r, f)$ and since $N_{(2}(r, 0 ; f)+N(r, \infty ; f)=$ $S(r, f)$, we have $\Phi_{1} \in S(f)$. From (20) we obtain

$$
f^{\prime \prime}(z)=\alpha_{1}(z) f(z)+\beta_{1}(z) f^{\prime}(z)
$$

where

$$
\alpha_{1}=\frac{\Phi_{1}}{P(f)} \text { and } \beta_{1}=K_{1} \frac{P^{\prime}(f)}{P(f)}-K_{2} .
$$

We note that $(21)$ is also true even for $\Phi_{1}(z) \equiv 0$. Actually in this case $\alpha_{1}(z) \equiv 0$. Also (22) yields

$$
P^{\prime}(f)=\left(\frac{\beta_{1}}{K_{1}}+\frac{K_{2}}{K_{1}}\right) P(f)
$$

and so

$$
\beta_{1}=K_{1} \frac{P^{\prime}(f)}{P(f)}-K_{2}=\frac{-k(k+1) !}{A_{1}} \frac{P^{\prime}(f)}{P(f)}-\frac{B_{1}}{A_{1}}
$$

i.e.,

$$
A_{1} \beta_{1}+B_{1}+k(k+1) ! \frac{P^{\prime}(f)}{P(f)}=0 .
$$

Now we consider following two subcases.

Subcase 1.2.1. Suppose $e^{\gamma} \in S(f)$. Now from (5) we have

$$
F^{(k)}-\alpha e^{\gamma} F=a_{2}-a_{1} \alpha e^{\gamma}+\beta e^{\gamma} .
$$

Suppose $a_{2}-a_{1} \alpha e^{\gamma}+\beta e^{\gamma} \not \equiv 0$. Since $n=k+1$, from $(25)$ we have $N_{1)}(r, 0 ; f)=S(r, f)$. Therefore from (18) we arrive to a contradiction. Therefore $a_{2}-a_{1} \alpha e^{\gamma}+\beta e^{\gamma} \equiv 0$ and so from (25) we get

$$
F^{(k)} \equiv \frac{a_{2} \alpha}{a_{1} \alpha-\beta} F
$$

which is the desired result.

Subcase 1.2.2. Suppose $e^{\gamma} \notin S(f)$. Following two subcases are immediately.

Subcase 1.2.2.1. Suppose $k=1$. Now from (16) and (21) we have

$$
P(f)=-2\left(f^{\prime}\right)^{2}-2 \xi f f^{\prime}+2 f f^{\prime \prime}=-2\left(f^{\prime}\right)^{2}+\left(2 \beta_{1}-2 \xi\right) f f^{\prime}+2 \alpha_{1} f^{2}
$$


and so

$$
P^{\prime}(f)=\left(-2 \beta_{1}-2 \xi\right)\left(f^{\prime}\right)^{2}+\left(2 \beta_{1}^{\prime}-2 \xi^{\prime}+2 \beta_{1}^{2}-2 \beta_{1} \xi\right) f f^{\prime}+\left(2 \alpha_{1} \beta_{1}-2 \alpha_{1} \xi+2 \alpha_{1}^{\prime}\right) f^{2} .
$$

Note that $K_{1}=1$ and $K_{2}=\xi$ and so from (23) we have

$$
\left(\beta_{1}^{\prime}-\xi^{\prime}-\beta_{1} \xi+\xi^{2}\right) f^{\prime}+\left(-2 \alpha_{1} \xi+\alpha_{1}^{\prime}\right) f \equiv 0 .
$$

We claim that $\beta_{1}^{\prime}-\xi^{\prime}-\beta_{1} \xi+\xi^{2} \not \equiv 0$. If not, suppose

$$
\beta_{1}^{\prime}-\xi^{\prime}-\beta_{1} \xi+\xi^{2} \equiv 0 .
$$

Let $\beta_{1} \equiv \xi$. Then a simple calculation gives $2\left(\frac{\alpha^{\prime}}{\alpha}+\gamma^{\prime}\right)=\frac{P^{\prime}(f)}{P(f)}$ and so on integration we get $\alpha^{2} e^{2 \gamma}=d_{0} P(f)$, where $d_{0} \in \mathbb{C} \backslash\{0\}$. This shows that $e^{\gamma} \in S(f)$, which is a contradiction. So $\beta_{1} \not \equiv \xi$. Now from (27) we get $\frac{\beta_{1}^{\prime}-\xi^{\prime}}{\beta_{1}-\xi}=\xi=\frac{\alpha^{\prime}}{\alpha}+\gamma^{\prime}$. So on integration we get $\alpha e^{\gamma}=d_{1}\left(\beta_{1}-\xi\right)$, where $d_{1} \in \mathbb{C} \backslash\{0\}$. This contradicts the fact that $e^{\gamma} \notin S(f)$. So $\beta_{1}^{\prime}-\xi^{\prime}-\beta_{1} \xi+\xi^{2} \not \equiv 0$. Since $f f^{\prime} \not \equiv 0$, from (27) we conclude that $-2 \alpha_{1} \xi+\alpha_{1}^{\prime} \not \equiv 0$. Then from (26) we see that if $z_{3}$ is a simple zero of $f$, then $z_{3}$ is either a pole of $-2 \alpha_{1} \xi+\alpha_{1}^{\prime}$ or a zero of $\beta_{1}^{\prime}-\xi^{\prime}-\beta_{1} \xi+\xi^{2}$. Hence

$$
N_{1)}(r, 0 ; f) \leq N\left(r, \infty ;-2 \alpha_{1} \xi+\alpha_{1}^{\prime}\right)+N\left(r, 0 ; \beta_{1}^{\prime}-\xi^{\prime}-\beta_{1} \xi+\xi^{2}\right)=S(r, f) .
$$

So we arrive to a contradiction by (18).

Subcase 1.2.2.2. Suppose $k \geq 2$. From (12) and (14) we have

$$
\begin{gathered}
F^{(k)}=T_{1}(f), F^{(k+1)}=(k+1) !\left(f^{\prime}\right)^{k+1}+T_{2}(f), \\
F^{(k+2)}=\frac{(k+1)(k+2)}{2}(k+1) !\left(f^{\prime}\right)^{k} f^{\prime \prime}+T_{3}(f),
\end{gathered}
$$

where $T_{1}(f), T_{2}(f)$ and $T_{3}(f)$ are differential polynomials in $f$ such that each term of $T_{1}(f)$, $T_{2}(f)$ and $T_{3}(f)$ contains $f$ as a factor. Comparing (11) and (14) and noting that $F=f^{n}=$ $f^{k+1}$ we have

$$
\begin{gathered}
\alpha Q(f)=\left(a_{1} \alpha-\beta\right) F^{(k+1)}-\left(a_{1} \alpha^{\prime}+a_{1}^{\prime} \alpha-\beta^{\prime}+a_{1} \alpha \gamma^{\prime}-\beta \gamma^{\prime}\right) F^{(k)}-a_{2} \alpha F^{\prime}+ \\
+\left(a_{2}^{\prime} \alpha-a_{2} \alpha \gamma^{\prime}-a_{2} \alpha^{\prime}\right) F+\delta=\left(a_{1} \alpha-\beta\right)\left\{(k+1) !\left(f^{\prime}\right)^{k+1}+T_{2}(f)\right\} \\
-\left(a_{1} \alpha^{\prime}+a_{1}^{\prime} \alpha-\beta^{\prime}+a_{1} \alpha \gamma^{\prime}-\beta \gamma^{\prime}\right) T_{1}(f)-(k+1) a_{2} \alpha f^{k} f^{\prime}+\left(a_{2}^{\prime} \alpha-a_{2} \alpha \gamma^{\prime}-a_{2} \alpha^{\prime}\right) f^{k+1}+\delta,
\end{gathered}
$$

where $\delta=a_{1} a_{2} \alpha \gamma^{\prime}+a_{1} a_{2} \alpha^{\prime}-a_{1} a_{2}^{\prime} \alpha+a_{1}^{\prime} a_{2} \alpha-a_{2} \beta \gamma^{\prime}-a_{2} \beta^{\prime}+a_{2}^{\prime} \beta$. First, we claim that $a_{1} \alpha-\beta \not \equiv 0$. If not, suppose $a_{1} \alpha-\beta \equiv 0$. In this case $\delta \equiv 0$. Then from (15) and (28) we have

$$
\left(a_{1} \alpha^{\prime}+a_{1}^{\prime} \alpha-\beta^{\prime}+a_{1} \alpha \gamma^{\prime}-\beta \gamma^{\prime}\right) F^{(k)}+a_{2} \alpha F^{\prime}-\left(a_{2}^{\prime} \alpha-a_{2} \alpha \gamma^{\prime}-a_{2} \alpha^{\prime}-\alpha P(f)\right) F \equiv 0,
$$

where $a_{2} \alpha \not \equiv 0$. Since $n=k+1$, from $(29)$ we have $N_{1)}(r, 0 ; f)=S(r, f)$. Therefore from (18) we arrive to a contradiction. Hence $a_{1} \alpha-\beta \not \equiv 0$.

Next we claim that $\delta(z) \not \equiv 0$. If not, suppose $\delta(z) \equiv 0$. Then from (15) and (28) we have

$$
\begin{gathered}
\left(a_{1} \alpha-\beta\right)\left\{(k+1) !\left(f^{\prime}\right)^{k+1}+T_{2}(f)\right\}-\left(a_{1} \alpha^{\prime}+a_{1}^{\prime} \alpha-\beta^{\prime}+a_{1} \alpha \gamma^{\prime}-\beta \gamma^{\prime}\right) T_{1}(f)- \\
-(k+1) a_{2} \alpha f^{k} f^{\prime}+\left(a_{2}^{\prime} \alpha-a_{2} \alpha \gamma^{\prime}-a_{2} \alpha^{\prime}-\alpha P(f)\right) f^{k+1} \equiv 0,
\end{gathered}
$$


Now from (30) we see that a simple zero of $f$ must be either a zero of $a_{1} \alpha-\beta$ or a pole of at least one of $a_{1} \alpha^{\prime}+a_{1}^{\prime} \alpha-\beta^{\prime}+a_{1} \alpha \gamma^{\prime}-\beta \gamma^{\prime}, \alpha, a_{2}^{\prime} \alpha-a_{2} \alpha \gamma^{\prime}-a_{2} \alpha^{\prime}-\alpha P(f)$. Therefore

$$
\begin{aligned}
& N_{1)}(r, 0 ; f) \leq N\left(r, 0 ; a_{1} \alpha-\beta\right)+N\left(r, \infty ; a_{1} \alpha^{\prime}+a_{1}^{\prime} \alpha-\beta^{\prime}+a_{1} \alpha \gamma^{\prime}-\beta\right) \leq \\
& \quad \leq N(r, \infty ; \alpha)+N\left(r, \infty ; a_{2}^{\prime} \alpha-a_{2} \alpha \gamma^{\prime}-a_{2} \alpha^{\prime}-\alpha P(f)\right) \leq S(r, f) .
\end{aligned}
$$

So we arrive to a contradiction from (18). Hence $\delta(z) \not \equiv 0$. We further note that $\delta \in S(f)$. Differentiating (28) we have

$$
\begin{gathered}
(\alpha Q(f))^{\prime}=\left(a_{1}^{\prime} \alpha+a_{1} \alpha^{\prime}-\beta^{\prime}\right) F^{(k+1)}+\left(a_{1} \alpha-\beta\right) F^{(k+2)}- \\
-\left(a_{1} \alpha^{\prime}+a_{1}^{\prime} \alpha-\beta^{\prime}+a_{1} \alpha \gamma^{\prime}-\beta \gamma^{\prime}\right) F^{(k+1)}- \\
-\left(a_{1} \alpha^{\prime}+a_{1}^{\prime} \alpha-\beta^{\prime}+a_{1} \alpha \gamma^{\prime}-\beta \gamma^{\prime}\right)^{\prime} F^{(k)}-\left(a_{2} \alpha\right)^{\prime} F^{\prime}-a_{2} \alpha F^{\prime \prime}+ \\
+\left(a_{2}^{\prime} \alpha-a_{2} \alpha \gamma^{\prime}-a_{2} \alpha^{\prime}\right)^{\prime} F+\left(a_{2}^{\prime} \alpha-a_{2} \alpha \gamma^{\prime}-a_{2} \alpha^{\prime}\right) F^{\prime}+\delta^{\prime}= \\
=\left(a_{1}^{\prime} \alpha+a_{1} \alpha^{\prime}-\beta^{\prime}\right)\left\{(k+1) !\left(f^{\prime}\right)^{k+1}+T_{2}(f)\right\}+ \\
+\left(a_{1} \alpha-\beta\right)\left\{\frac{(k+1)(k+2)}{2}(k+1) !\left(f^{\prime}\right)^{k} f^{\prime \prime}+T_{3}(f)\right\}- \\
-\left(a_{1} \alpha^{\prime}+a_{1}^{\prime} \alpha-\beta^{\prime}+a_{1} \alpha \gamma^{\prime}-\beta \gamma^{\prime}\right)\left\{(k+1) !\left(f^{\prime}\right)^{k+1}+T_{2}(f)\right\}- \\
-\left(a_{1} \alpha^{\prime}+a_{1}^{\prime} \alpha-\beta^{\prime}+a_{1} \alpha \gamma^{\prime}-\beta \gamma^{\prime}\right)^{\prime} T_{1}(f)-(k+1)\left(a_{2} \alpha\right)^{\prime} f^{k} f^{\prime}- \\
-a_{2} \alpha\left\{k(k+1) f^{k-1}\left(f^{\prime}\right)^{2}+(k+1) f^{k} f^{\prime \prime}\right\}+\left(a_{2}^{\prime} \alpha-a_{2} \alpha \gamma^{\prime}-a_{2} \alpha^{\prime}\right)^{\prime} f^{k+1}+ \\
+(k+1)\left(a_{2}^{\prime} \alpha-a_{2} \alpha \gamma^{\prime}-a_{2} \alpha^{\prime}\right) f^{k} f^{\prime}+\delta^{\prime} .
\end{gathered}
$$

Let $z_{4}$ be a simple zero of $f(z)$. Then from (15), (28) and (31) we have

$$
\delta\left(z_{4}\right)=A\left(z_{4}\right)\left(f^{\prime}\left(z_{4}\right)\right)^{k+1}, \quad \delta^{\prime}\left(z_{4}\right)=A_{2}\left(z_{4}\right)\left(f^{\prime}\left(z_{4}\right)\right)^{k} f^{\prime \prime}\left(z_{4}\right)+B_{2}\left(z_{4}\right)\left(f^{\prime}\left(z_{4}\right)\right)^{k+1},
$$

where

$$
\begin{gathered}
A(z)=-(k+1) !\left(a_{1}(z) \alpha(z)-\beta(z)\right), \quad A_{2}(z)=-\frac{(k+1)(k+2)}{2}(k+1) !\left(a_{1}(z) \alpha(z)-\beta(z)\right), \\
B_{2}(z)=(k+1) !\left(a_{1}(z) \alpha(z)-\beta(z)\right) \gamma^{\prime}(z) .
\end{gathered}
$$

This shows that $z_{4}$ is a zero of $\delta f^{\prime \prime}-\left[K_{3} \delta^{\prime}-K_{4} \delta\right] f^{\prime}$, where $K_{3}=\frac{A}{A_{2}}$ and $K_{4}=\frac{B_{2}}{A_{2}}$. Also we see that $K_{3} \in S(f)$ and $K_{4} \in S(f)$. Let

$$
\Phi_{2}=\frac{\delta f^{\prime \prime}-\left[K_{3} \delta^{\prime}-K_{4} \delta\right] f^{\prime}}{f} .
$$

Suppose $\Phi_{2}(z) \not \equiv 0$. Then clearly $m\left(r, \Phi_{2}\right)=S(r, f)$ and since $N_{(2}(r, 0 ; f)+N(r, \infty ; f)=$ $S(r, f)$, we have $\Phi_{2} \in S(f)$. From (32) we obtain

$$
f^{\prime \prime}=\phi_{1} f+\psi_{1} f^{\prime}
$$

where

$$
\phi_{1}=\frac{\Phi_{2}}{\delta} \text { and } \psi_{1}=K_{3} \frac{\delta^{\prime}}{\delta}-K_{4}
$$


We note that (33) is also true even for $\Phi_{2}(z) \equiv 0$. Actually in this case $\phi_{1}(z) \equiv 0$.

Now we claim that $\psi_{1} \not \equiv \beta_{1}$. If $\psi_{1} \equiv \beta_{1}$ then from (22) and (34) we have i.e.,

$$
\frac{2}{(k+1)(k+2)} \frac{\delta^{\prime}}{\delta}+\frac{2}{(k+1)(k+2)} \gamma^{\prime} \equiv \frac{4}{(k+1)^{2}} \frac{P(f)^{\prime}}{P(f)}-\frac{4}{k(k+1)^{2}} \xi,
$$

On integration we have

$$
2 k(k+2) \frac{P(f)^{\prime}}{P(f)}-k(k+1) \frac{\delta^{\prime}}{\delta} \equiv\left(k^{2}+3 k+4\right) \gamma^{\prime}+2(k+2) \frac{\alpha^{\prime}}{\alpha} .
$$

$$
\alpha^{2(k+2)} e^{\left(k^{2}+3 k+4\right) \gamma} \equiv \frac{d_{3} P(f)^{2 k(k+2)}}{\delta^{k(k+1)}}
$$

where $d_{3} \in \mathbb{C} \backslash\{0\}$ and so from (17) we have $e^{\gamma} \in S(f)$, a contradiction. So we suppose that $\psi_{1} \not \equiv \beta_{1}$.

Now from (33) we have

$$
f^{(i)}=\phi_{i-1} f+\psi_{i-1} f^{\prime}
$$

where $i \geq 2$ and $\phi_{i-1} \in S(f), \psi_{i-1} \in S(f)$. Also from (16), (19) and (35) we have respectively

$$
\begin{gathered}
P(f)=-k(k+1) !\left(f^{\prime}\right)^{k+1}+\sum_{j=1}^{k+1} T_{j} f^{j}\left(f^{\prime}\right)^{k+1-j} \\
P^{\prime}(f)=\left(A_{1} \psi_{1}+B_{1}\right)\left(f^{\prime}\right)^{k+1}+\sum_{j=1}^{k+1} S_{j} f^{j}\left(f^{\prime}\right)^{k+1-j},
\end{gathered}
$$

where $T_{j} \in S(f)$ and $S_{j} \in S(f)$. Multiplying (36) by $P^{\prime}(f)$ and $(37)$ by $P(f)$ and then subtracting we get

$$
H_{0}\left(f^{\prime}\right)^{k+1}+H_{1} f\left(f^{\prime}\right)^{k}+\ldots+H_{k+1} f^{k+1} \equiv 0,
$$

where

$$
H_{0}=P(f)\left[A_{1} \psi_{1}+B_{1}+k(k+1) ! \frac{P^{\prime}(f)}{P(f)}\right]
$$

and $H_{j}=P(f) S_{j}-P^{\prime}(f) T_{j}$ for $j=1,2, \ldots, k+1$. Note that $H_{0} \in S(f)$ and $H_{j} \in S(f)$ for $j=1,2, \ldots, k+1$. Since $\beta_{1} \not \equiv \psi_{1}$, it follows from (24) and (39) that $H_{0} \not \equiv 0$. Now from (38) we see that a simple zero of $f$ must be either a zero of $H_{0}$ or a pole of at least one of $H_{1}, H_{2}, \ldots H_{k+1}$. Therefore

$$
N_{1)}(r, 0 ; f) \leq N\left(r, 0 ; H_{0}\right)+\sum_{i=1}^{k+1} N\left(r, \infty ; H_{i}\right)+S(r, f)=S(r, f),
$$

because $H_{i} \in S(f)$ for $i=0,1,2, \ldots, k+1$. So we arrive to a contradiction from (18).

Case 2. Suppose $F^{(k+1)} F-\xi F^{(k)} F-F^{(k)} F^{\prime} \equiv 0$. Then we have

$$
\frac{F^{(k+1)}}{F^{(k)}} \equiv \frac{\alpha^{\prime}}{\alpha}+\gamma^{\prime}+\frac{F^{\prime}}{F}
$$

On integration, we have $F^{(k)}=d_{4} \alpha e^{\gamma} F$, where $d_{4} \in \mathbb{C} \backslash\{0\}$. Now from (5) we have

$$
\left(d_{4}-1\right) \alpha e^{\gamma} f^{n} \equiv a_{2}-\left(a_{1} \alpha-\beta\right) e^{\gamma} .
$$


If $d_{4}=1$, then from (40) we have $a_{2}-\left(a_{1} \alpha-\beta\right) e^{\gamma} \equiv 0$. Now from (5) we get $F^{(k)} \equiv \frac{a_{2} \alpha}{a_{1} \alpha-\beta} F$, which is the desired result.

Next we suppose $d_{4} \neq 1$. Now we consider following two subcases.

Subcase 2.1. Suppose $e^{\gamma} \in S(f)$. Clearly from (40) and Lemma 3 we have $n T(r, f)=$ $S(r, f)$, which is a contradiction.

Subcase 2.2. Suppose $e^{\gamma} \notin S(f)$. Now from (40) we have $e^{-\gamma} \equiv\left(d_{4}-1\right) \frac{\alpha}{a_{2}} f^{n}+\frac{1}{a_{2}}\left(a_{1} \alpha-\beta\right)$. By Lemma 3 we get

$$
T\left(r, e^{\gamma}\right)=T\left(r, e^{-\gamma}\right)+O(1)=n T(r, f)+S(r, f)
$$

and so $S(r, f)=S\left(r, e^{\gamma}\right)$. Therefore $a_{1}, a_{2}, \alpha, \beta \in S\left(e^{\gamma}\right)$. Also from (40) we have

$$
\bar{N}\left(r, \frac{a_{2}}{a_{1} \alpha-\beta} ; e^{\gamma}\right) \leq \frac{1}{n} N\left(r, \frac{a_{2}}{a_{1} \alpha-\beta} ; e^{\gamma}\right) \leq \frac{1}{n} T\left(r, e^{\gamma}\right) .
$$

Now by Lemma 2 we have

$$
T\left(r, e^{\gamma}\right) \leq \bar{N}\left(r, \infty ; e^{\gamma}\right)+\bar{N}\left(r, 0 ; e^{\gamma}\right)+\bar{N}\left(r, \frac{a_{2}}{a_{1} \alpha-\beta} ; e^{\gamma}\right)+S\left(r, e^{\gamma}\right) \leq \frac{1}{n} T\left(r, e^{\gamma}\right)+S\left(r, e^{\gamma}\right),
$$

which is impossible since $n \geq k+1 \geq 2$.

\section{REFERENCES}

1. Brück R., On entire functions which share one value $C M$ with their first derivative, Results Math., 30 (1996), 21-24.

2. Cao T.B., On the Brück conjecture, Bull. Aust. Math. Soc., 93 (2016), 248-259.

3. Chen Z.X., Shon K.H., On conjecture of R. Brück concerning the entire function sharing one value $C M$ with its derivative, Taiwanese J. Math., 8 (2004), №2, 235-244.

4. Clunie J., On integral and meromorphic functions, J. London Math. Soc., 37 (1962), 17-22.

5. Gundersen G.G., Yang L.Z., Entire functions that share one value with one or two of their derivatives, J. Math. Anal. Appl., 223 (1998), 88-95.

6. Hayman W.K., Meromorphic Functions, Clarendon Press, Oxford, 1964.

7. Lü F., Yi H.X., The Brück conjecture and entire functions sharing polynomials with their $k$-th derivatives, J. Korean Math. Soc., 48 (2011), №3, 499-512.

8. Lü F., Li Q., Yang C., On the transcendental entire solutions of a class of differential equations, Bull. Korean Math. Soc., 51 (2014), №5, 1281-1289.

9. Yang L.Z., Zhang J.L., Non-existence of meromorphic solutions of Fermat type functional equation, Aequations Math., 76 (2008), №1-2, 140-150.

10. Yang C.C., On deficiencies of differential polynomials II, Math. Z., 125 (1972), 107-112.

11. Zhang J.L., Meromorphic functions sharing a small function with their derivatives, Kyungpook Math. J., 49 (2009), 143-154.

12. Zhang J.L., Yang L.Z., A power of a meromorphic function sharing a small function with its derivative, Annales Academiæ Scientiarum Fennicæ Mathematica, 34 (2009), 249-260.

13. Zhang J.L., Yang L.Z., A power of an entire function sharing one value with its derivative, Comput. Math. Appl, 60 (2010), 2153-2160.

Department of Mathematics, Raiganj University

Raiganj, West Bengal, India

sujoy.katwa@gmail.com

sm05math@gmail.com

smajumder05@yahoo.in 